\title{
Characteristic Features of the Pseudogap and Superconducting States of $\mathrm{YBa}_{2} \mathrm{Cu}_{3} \mathrm{O}_{7-x}$
}

\author{
O. V. Misochko*, N. Georgiev**, T. Dekorsy**, and M. Helm** \\ * Institute of Solid State Physics, Russian Academy of Sciences, Chernogolovka, Moscow region, 142432 Russia \\ ** Institute for Ion Beam Physics and Materials Research, D-01314 Dresden, Germany \\ e-mail: misochko@issp.ac.ru
}

\begin{abstract}
The relaxation dynamics of the lattice and low-energy quasiparticles in a $\mathrm{YBa}_{2} \mathrm{Cu}_{3} \mathrm{O}_{7-x}$ superconductor are studied by the light reflection technique with femtosecond temporal resolution in a wide temperature range. It is shown that, for $T>T_{c}$, there exist two temperature regions with qualitatively and quantitatively different excitation dynamics, and the transition between these regions is of a hysteretic nature. It is also found that the character of changes observed in the charge carrier relaxation dynamics in the superconducting state testifies to the presence of an anisotropic gap with nodes at the Fermi surface.
\end{abstract}

For the past few years, one of the major problems in high-temperature superconductivity (HTSc) has been the study of the physics of the pseudogap state, which exists in the metallic phase at temperatures $T^{*}>T_{c}[1$, 2]. Today, this problem seems to be among the most topical ones in the physics of high-temperature superconductivity, and its solution will undoubtedly contribute to the elucidation of the microscopic mechanism of HTSc. The width of the pseudogap state region $T^{*}-T_{c}$ in the phase diagram depends on the carrier concentration. It is maximal for underdoped compounds and decreases to zero at a certain critical carrier concentration; the latter is somewhat higher than the concentration at which the critical temperature $T_{c}$ is maximal. Recent theoretical papers suggest an inhomogeneity of the pseudogap phase and the existence of the temperature crossover, which separates the regions of the pseudogap states with different dynamic properties of quasiparticles [3-5]. Progress in laser technology has made it possible to decrease the laser pulse duration down to a few femtoseconds, and this has opened up new research prospects by making studies of quasiparticle dynamics and lattice vibrations accessible in real time. Such studies, whose typical example is the pumping technique with subsequent probing by two laser pulses separated in time, are actively performed on HTSc systems [6-9]. The understanding of the mechanism underlying the establishment of equilibrium after its perturbation by a laser pulse gives an insight into the characteristic features of the dynamics of charge carriers and crystal lattice excitations, as well as the dynamics of their interaction. Using the pumping-probing technique, we performed detailed studies of the relaxation dynamics of the lattice and charge carriers in the temperature range covering both superconducting and pseudogap states. The aim of our studies was to determine the degree of homogeneity of the phase diagram region for $T>T_{c}$ and to reveal the characteristic features of the superconducting state.

The studies of the optical response were performed on an optimally doped $\left(T_{c}=88 \mathrm{~K}\right)$ epitaxial film of $\mathrm{YBa}_{2} \mathrm{Cu}_{3} \mathrm{O}_{7-x}(\mathrm{Y} 123)$ grown on a $\mathrm{SrTiO}_{3}$ substrate. The film thickness was $350 \mathrm{~nm}$, and its $c$ axis was perpendicular to the substrate plane. The sample was placed in an optical helium cryostat, which allowed the measurements in the temperature range 4-310 K. The measurements in the time domain were done using a fast scanning system [6] and sapphire titanate laser pulses $(\lambda=780 \mathrm{~nm})$ with a duration of less than $50 \mathrm{fs}$ and a repetition frequency of $78 \mathrm{MHz}$. The polarizations of the exciting and probing pulses were orthogonal to each other and lay in the film plane. In the experiment, the differential reflection from the excited and nonexcited samples, $\Delta R(t)=R(t)-R_{0}$, was measured as a function of time $t$ between pumping and probing in a wide temperature range.

Figure 1 shows a typical optical response obtained from the excitation and subsequent probing of the Y123 film by the femtosecond pulses. The excited electron state relaxes to the equilibrium state within the time of order of picoseconds. The decay of the photoinduced differential reflection has a non-exponential character and may be described by a sum of two, fast and slow, exponentials: $\Delta R / R_{0}=A(T) \exp \left(-t / \tau_{A}\right)+B(T) \exp \left(-t / \tau_{B}\right)$. Fast oscillations shown in the inset in Fig. 1 and associated with the coherent phonons $[6,7]$ are superimposed on this electronic decay. For the identification of the phonon modes, the data obtained in the time domain were numerically transformed to the frequency domain by using the fast Fourier transform. This paper studies 
both the oscillatory component of the optical response caused by the excitation of coherent optical phonons and the nonoscillatory response related to the charge carrier relaxation.

In the superconducting state, two fully symmetric phonon modes generated by the corresponding displacements of the $\mathrm{Ba}$ and $\mathrm{Cu}$ ions (see Fig. 2) [6,7] are detected in the spectrum of coherent phonons. An increase in temperature leads to a decrease in the amplitude of the $\mathrm{Ba}$ mode. Therefore, the $\mathrm{Cu}$ mode dominates in the spectrum in the vicinity of the superconducting transition, which can be easily traced by comparing the amplitudes of the coherent phonons related to $\mathrm{Ba}$ and $\mathrm{Cu}$. At helium temperatures, the electron relaxation is governed by the slow component, which demonstrates a weak singularity at $T_{c}$. The quantity $\Delta R / R_{0}$ continues decreasing above $T_{c}$; however, at temperatures above $T>T_{1}^{*}=160 \mathrm{~K}$, the photoinduced response abruptly changes its behavior. The fast component of the electron relaxation exhibits a change of sign and reduces to a peak whose duration does not exceed that of the laser pulse. The slow component also changes sign and considerably slows down. Simultaneously, the spectrum of coherent phonons changes, becoming similar to the spectrum observed in the superconducting state. A further increase in temperature above $T_{2}^{*}=220 \mathrm{~K}$ results in the recovery of the character of the photoinduced response observed in the temperature range $T_{c}-T_{1}^{*}$. Interestingly, the temperatures $T_{1}^{*}$ and $T_{2}^{*}$ corresponding to sharp changes in the relaxation dynamics of charge carriers and crystal lattice exhibit a hysteretic character. When approaching the superconducting transition from above, the changes in the photoinduced response are observed at $T_{2}^{*}=175 \mathrm{~K}$ and $T_{1}^{*}=115 \mathrm{~K}$. The results obtained for the relaxation dynamics of the charge carriers and lattice are summarized in Fig. 3.

The idea of the inhomogeneity of the pseudogap state of HTSc was deduced theoretically and may be explained as a consequence of the local pairing and delocalization of the electron pairs [5]. In addition, the inhomogeneous state may originate from weak and strong regimes of the almost antiferromagnetic Fermi liquid [4] or from the formation of a charge inhomogeneity (stripe fluctuations) with the subsequent appearance of superconductivity in individual stripes [3]. The results obtained do not allow one to give preference to either of the above models. First of all, this is related to the fact that the hysteretic behavior has been predicted by none of the models; it still remains unclear whether different models differ from each other and have any fundamental limitations in this respect. However, certain features of the relaxation dynamics may be explained by each theory. For example, the acceleration of the fast component and deceleration of the slow

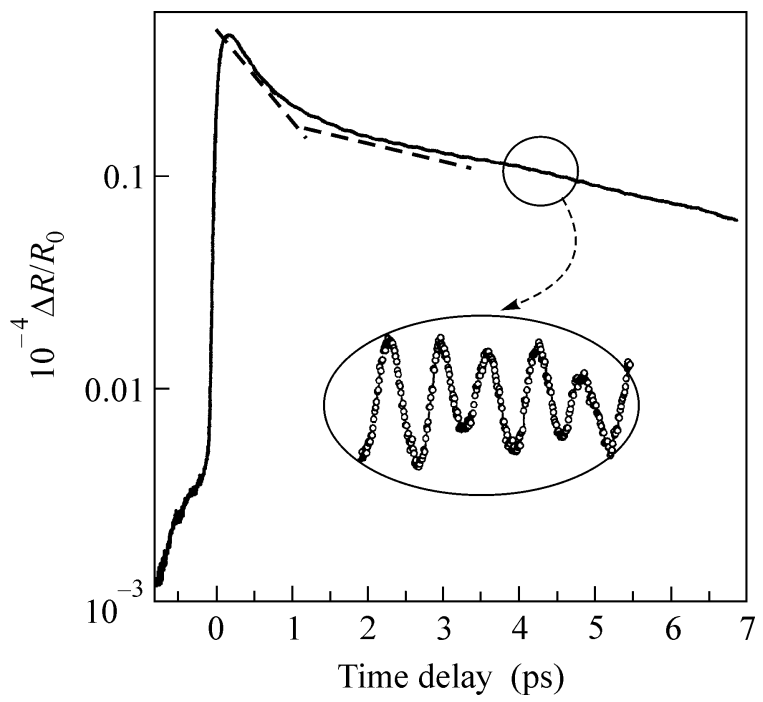

Fig. 1. Time-resolved differential reflection $\Delta R / R_{0}$ (on a logarithmic scale) for $\mathrm{YBa}_{2} \mathrm{Cu}_{3} \mathrm{O}_{7-x}$ at room temperature. The dashed lines show the fast and slow relaxation channels. The inset magnifies the oscillations caused by the coherent phonon excitation.

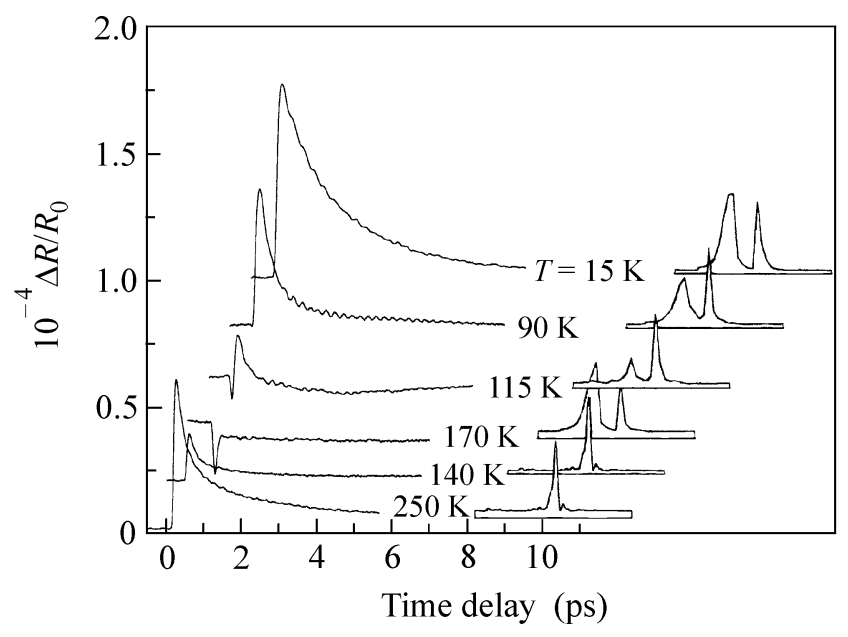

Fig. 2. Temperature dependence of the time-resolved differential reflection $\Delta R / R_{0}$. The Fourier transforms of the oscillatory parts are shown on the right of each transient curve. The two pronounced peaks at frequencies of 3.5 and $4.5 \mathrm{THz}$ correspond to the fully symmetric $A_{g}$ phonons generated by the $\mathrm{Ba}$ and $\mathrm{Cu}$ ion displacements.

component within the temperature range $T_{1}^{*}-T_{2}^{*}$ may be evidence for the appearance of local short-lived electron pairs whose constituents are localized. The disappearance of (decrease in) the slow component at $T<T_{1}^{*}$ may be interpreted as the beginning of the electron pair motion over the crystal. The similarity of the coherent 


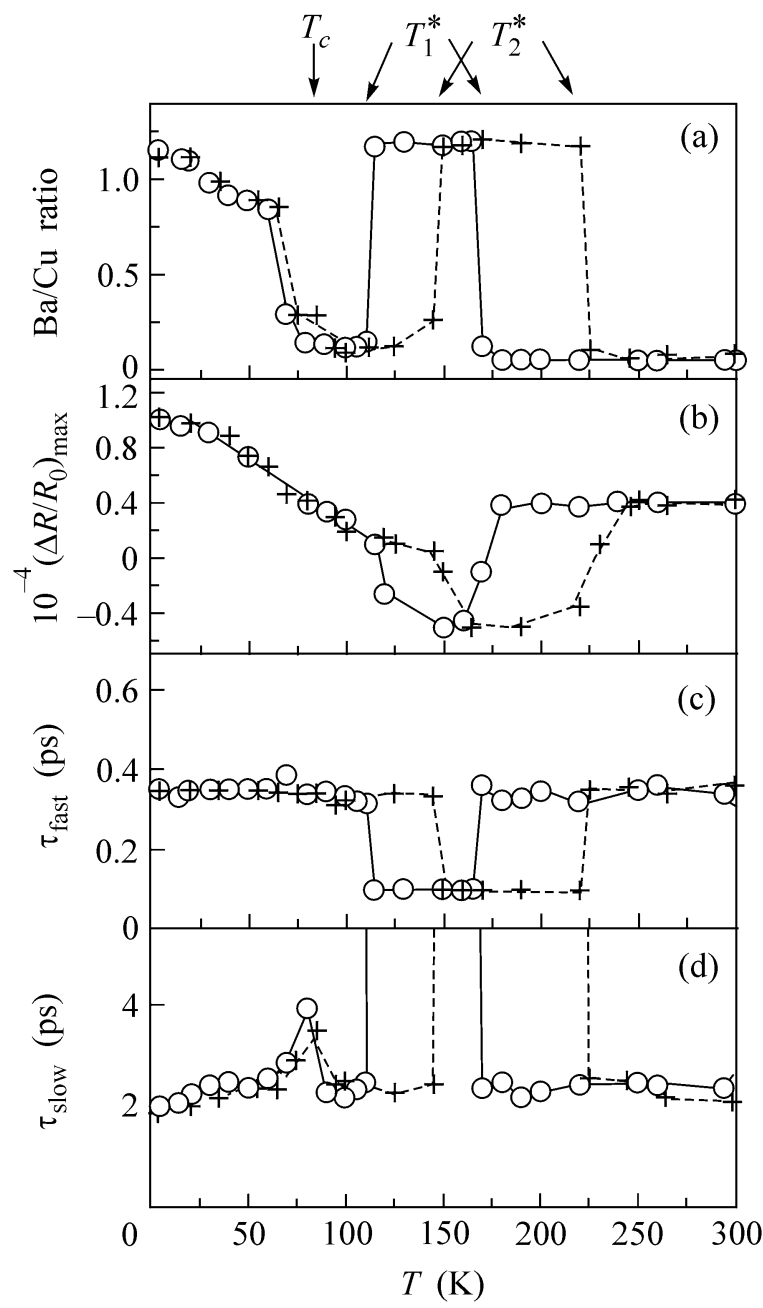

Fig. 3. Parameters of the relaxation dynamics of the excitations and lattice versus the temperature. The circles correspond to the data obtained with decreasing temperature, and the crosses, with increasing temperature. (a) Ratio of the phonon amplitudes of the $\mathrm{Ba}$ and $\mathrm{Cu}$ modes in the periodogram; (b) extremum of the differential reflection $\left(\Delta R / R_{0}\right)_{\max } ;$ (c) fast relaxation time; and (d) slow relaxation time.

phonon spectra for the temperature ranges $T_{1}^{*}-T_{2}^{*}$ and $T<T_{c}$ also supports the hypothesis of the formation of local electron pairs, because the increase in the $\mathrm{Ba}$ phonon amplitude is usually attributed to the breaking of Cooper pairs [6, 7]. However, in the range $T_{1}^{*}-T_{c}$, where, according to the theory in [5], the pairs already move over the crystal, the spectrum of coherent phonons exhibits a similarity to the spectrum observed at temperatures above the second crossover $T_{2}^{*}$. It remains unclear why the delocalized but still incoherent pairs cease interacting with the lattice, whereas the localized pairs and Cooper pairs, which constitute the superconducting condensate, demonstrate this kind of interaction. Nevertheless, the similarity of the coherent dynamics of the lattice in the pseudo-gap and supercon- ducting states enables one to assume that the electronphonon interaction is needed to explain the experimental data. As an alternative explanation, the upper crossover could be assigned to the beginning of the weak regime of the pseudogap state, when the hot spots start appearing at the Fermi surface. The lower crossover then could be attributed to the appearance of the strong regime, in which the Fermi surface starts loosing its parts in some regions of the momentum space [4]. However, the changes in the coherent phonon spectrum can hardly be explained in terms of an almost antiferromagnetic Fermi liquid.

In contrast to the pseudogap state, the superconducting state exhibits no hysteresis, and the characteristics of the relaxation dynamics coincide within the experimental error for both increasing and decreasing temperature. This result is quite natural, because the superconducting transition in the absence of a magnetic field is of second order, whereas the hysteresis is characteristic of the first-order transitions only. However, it should be noted that the ratio between the slow $(B)$ and fast $(A)$ components in the superconducting state is temperature-dependent (it increases as $T$ decreases). The fact that the slow component predominates in the superconducting state is determined by the appearance of the gap in the spectrum of excitations. Breaking the Cooper pairs with a laser pulse leads to the appearance of quasiparticles whose relaxation is determined by the relaxation of the superconducting gap to the equilibrium state. If we attribute the fast dynamics to the carriers in the cold spots, which are located at the diagonals of the Brillouin zone, and the slow dynamics, to the carriers in the vicinity of the hot spots, which are located at the zone faces where the superconducting gap takes its maximal values, we should expect that the superconducting gap continues breaking the Fermi surface in the superconducting state as well. Such an association of the two different dynamic times with the quasiparticle positions in the Brillouin zone is supported by the following experimental fact [10]: it was found that the dependence of the slow component on the pumping power exhibits a saturation, whereas no saturation is observed for the slow component, whose amplitude grows linearly with the power of the exciting pulses. This fact enables us to attribute the slow component to the breaking of Cooper pairs, whose density is finite in the condensate, and to assign the fast component to the quasiparticles in the vicinity of the nodes of the superconducting gap. The variation of the ratio $B / A$ in the superconducting state testifies to (1) the existence of nodes of the superconducting gap, because the fast component is finite at the lowest temperatures, and (2) the dependence of the anisotropy of the gap on temperature, which dramatically disagrees with the predictions of the Bardeen-Cooper-Schrieffer theory, in which the anisotropy of the gap is found to be temperature-independent as a result of the factorization of the wave vector and temperature in the equation for the superconducting gap [11]. The conclusion that an 
anisotropic superconducting gap with nodes at the Fermi surface exists agrees well with the experimental results obtained by other optical techniques [11], although it differs from the data on the gap obtained from earlier time-resolved measurements [8].

Thus, by using the pumping-probing technique with femtosecond resolution, we observed two temperature crossovers in the normal state of an optimally doped Y123 high-temperature superconductor. The crossovers manifest themselves as sharp changes in the relaxation dynamics of the charge carriers and the lattice and exhibit a hysteresis in temperature. The existence of such crossovers testifies to the inhomogeneity of the pseudogap state. In addition, we have found that, in the superconducting state, the energy gap has nodes and its anisotropy depends on temperature.

This work was supported by the Russian Foundation for Basic Research, project no. 01-02-1640.

\section{REFERENCES}

1. T. Timusk and B. Statt, Rep. Prog. Phys. 62, 61 (1999).

2. M. V. Sadovski1̌, Usp. Fiz. Nauk 171, 539 (2001).
3. V. J. Emery, S. A. Kivelson, and O. Zachar, Phys. Rev. B 56, 6120 (1997).

4. J. Schmalian, D. Pines, and B. Stojkovic, Phys. Rev. B 60, 667 (1999).

5. P. Devillard and J. Ranninger, Phys. Rev. Lett. 84, 5200 (2000).

6. W. Albrecht, Th. Kruze, and H. Kurz, Phys. Rev. Lett. 69, 1451 (1992).

7. O. V. Misochko, K. Kisoda, K. Sakai, and S. Nakashima, Phys. Rev. B 61, 4305 (2000).

8. C. J. Stevens, D. Smith, C. Chen, et al., Phys. Rev. Lett. 78, 2212 (1997).

9. J. Demsar, B. Podobnik, V. V. Kabanov, et al., Phys. Rev. Lett. 82, 4918 (1999).

10. R. A. Kaindl, M. Woerner, T. Elsaesser, et al., Science 287, 470 (2000); R. A. Kaindl, Ph.D. Dissertation (Humboldt University, Berlin, 2000).

11. E. Ya. Sherman, O. V. Misochko, and P. Lemmens, in Spectroscopy of High- $T_{c}$ Superconductors, Ed. by N. M. Plakida (Taylor \& Francis, London, 2002), p. 97.

Translated by E. Golyamina 